\title{
Actitudes de alumnos de posgrado hacia la estadística aplicada a la investigación
}

Eduardo Escalante Gómez

Director Instituto de Investigaciones, Facultad de Psicología, Universidad del Aconcagua, Mendoza, Argentina. Correo electrónico: escalante.gomez@gmail.com

\section{Recibido: diciembre de 2009 / Aceptado: marzo de 2010}

EN ESTA INVESTIGACIÓN SE EXAMINA SI EXISTEN CAMBIOS DE ACTITUDES EN LOS ALUMNOS de posgrado que participan en un módulo de estadística aplicado a la investigación. El autor investigó si los alumnos desarrollaron actitudes más positivas hacia la estadística después del desarrollo del módulo, que implicó una estrategia didáctica específica. Los resultados revelan que el desarrollo de las actividades que permiten el diseño de configuraciones didácticas, trayectorias didácticas, trayectorias epistémicas y trayectorias de mediación, teniendo como eje central la construcción del conocimiento estadístico por los propios alumnos, genera aprendizajes más significativos y actitudes más positivas hacia la estadística. Estas conclusiones tienen implicaciones importantes para preparar a los alumnos de posgrado en la elaboración de sus tesis o investigaciones.

Palabras clave: actitudes / estadística / configuraciones didácticas / trayectos didácticos / trayectos epistémicos

\section{Introducción}

La literatura revela que la habilidad para comprender, interpretar y evaluar críticamente los hallazgos de las investigaciones, tiene un impacto directo en el éxito de los alumnos de posgrado al momento de desarrollar sus tesis o investigaciones, por lo cual estas habilidades han pasado a estimarse como esenciales o nucleares. De especial importancia es abordar este tema porque los estudios de posgrado se basan fundamentalmente en la elaboración de tesis basadas en investigación empírica y existen evidencias sobre las dificultades de los alumnos de posgrado en el manejo de la estadística.

El estudio de las actitudes hacia la estadística no es nuevo, existen distintas aproximaciones conceptuales y una variedad de instrumentos de medición. Un estudio comprensivo y actualizado de la investigación empírica realizada sobre las actitudes de los alumnos universitarios hacia la Estadística es el de Blanco (2008): Una revisión crítica de la investigación sobre las actitudes de los estudiantes universitarios hacia la Estadística. El autor concluye que la línea de trabajo de Sorge y Schau (2002) representa un esfuerzo 
importante para generar referentes teóricos al momento de investigar las actitudes de los alumnos hacia la estadística. Schau, Stevens, Dauphine y Del Vecchio (1995), en especial Schau, ha venido trabajando en los procesos de medición de este tipo de actitudes mediante la escala SATS (Survey of Attitudes Toward Statistics). Sorge y Schau (2002) señalan que sus resultados son consistentes con un modelo de logro estadístico en el cual las actitudes hacia las estadísticas son causas importantes para el logro estadístico en estudiantes de ingeniería, aunque no tan importantes como los logros previos.

El propósito de esta investigación fue explorar en qué medida las actitudes hacia la estadística en alumnos de posgrado mejoraron mediante un enfoque basado en la elaboración de configuraciones didácticas y su articulación en trayectorias didácticas, trayectorias epistémicas y trayectorias de mediación.

\section{Revisión de la literatura}

Lo explicado en los párrafos anteriores se fundamenta en muchas de las investigaciones que, por una parte, han reunido evidencias empíricas sobre la presencia de actitudes negativas al momento de aprender estadística, y por otra, han señalado la necesidad de involucrar a los alumnos en procesos de "significar o interpretar", en especial, si participan de procesos reales, en este caso, procesos investigativos en el área de la ciencias sociales (Lewis \& Williams, 1994).

Una revisión de los componentes curriculares de los posgrados revela que los alumnos se ven involucrados en el estudio de la estadística, ya sea la descriptiva, la inferencial, o la multivariada exploratoria y confirmatoria, a la que actualmente muchos de ellos están siendo introducidos. En consecuencia, es común que los alumnos deban trabajar con conceptos como frecuencias relativas y frecuencias absolutas, medidas de tendencia central y medidas de variabilidad, tablas de contingencia, comparaciones de medias, diferencias de medias con dos o más grupos, análisis de correspondencias, análisis factorial, etcétera.

Las investigaciones han detectado que los contenidos de la estadística que presentan mayores problemas de aprendizaje son la comprensión de la probabilidad, la prueba de hipótesis, la distribución muestral, el análisis de varianza, el análisis de regresión, la correlación y el diseño experimental; a diferencia de las medidas de tendencia central y dispersión, que presentarían menos problemas de comprensión para los alumnos de posgrado.

La noción de actitud, muy utilizada en el terreno de la psicología social, dista de haber obtenido hasta el momento una caracterización universalmente aceptada. Se considera que las actitudes no son directamente observables sino que son el resultado de procesos de abstracción e inferencia relacionados con los sentimientos y predisposiciones de los individuos hacia los objetos con los que se relacionan. Para autores como Gal y Garfield (1997), las actitudes representan una sumatoria de emociones y sentimientos que se experimentan durante el período de aprendizaje de la materia objeto de estudio.

La revisión de la literatura permite destacar la conceptualización de las actitudes que reconoce la dimensión valorativa (Eagly \& Chaiken, 1993). Este tipo de enfoque es 
importante desde el punto de vista estructural en cuanto pone énfasis en las actitudes como estados internos de naturaleza evaluativa, entendidos como estados personales que sugieren la predisposición para actuar de una manera determinada. Para la perspectiva estructural, las actitudes se entienden como modelos de redes que se derivan de teorías de aprendizaje asociativo e introducen el componente de la memoria. Este tipo de enfoque puede ser enriquecido a partir de una contextualización sociocultural de las actitudes. Por ejemplo, asociarlas a los campos disciplinarios de origen de los alumnos de posgrado $u$ otras variables de corte socio-cultural.

El estudio de las actitudes y respuestas afectivas hacia la estadística ha sido informado por diferentes autores: Kirk (2002), Gal, Ginsburg y Schau (1997), entre otros. Para modificar las actitudes hacia la estadística se han estudiado las ventajas de que los alumnos participen en procesos activos de producción de datos y resultados (Cobb, 1993). La experiencia práctica indica que en la medida que los datos son relevantes para el proceso investigativo que deben realizar los alumnos, éstos se sienten más motivados e interesados para realizar los procesos de análisis estadísticos. Surgen preguntas que cada vez son más pertinentes y relevantes.

Se ha detectado que los alumnos que se involucran activamente en definir problemas y analizar datos tienen experiencias emocionales positivas al analizar el material estadístico (Bradstreet, 1996; Keeler \& Steinhorst, 1995; Romero et al., 1995). Existen evidencias que la enseñanza del análisis estadístico no se puede reducir a la mera mecánica del cálculo, sino que debe desarrollarse habilidades de comprensión del pensamiento estadístico (Snee, 1993; Gal \& Ginsburg, 1994; Watts, 1991).

Uno de los objetivos fundamentales del estudio de la estadística en los posgrados debería ser enseñar a los alumnos a pensar críticamente usando conceptos fundamentales de la estadística y su aplicación a problemas reales del campo disciplinario específico al que pertenecen (ingeniería, ciencias sociales, etc.). Los alumnos deberían ser capaces de organizar y resumir datos, formular inferencias a partir de los resúmenes estadísticos, e incorporarlos en sus informes. Los principios de aprendizaje activo, aprendizaje auténtico y aprendizaje colaborativo resultan relevantes.

Las investigaciones informan de actitudes negativas hacia la estadística, las cuales se pueden constituir en un obstáculo para los procesos de aprendizaje del proceso investigativo y, en general, para la efectividad del aprendizaje al momento de investigar (Schutz, Drogosz, White \& Distefano, 1998).

Existen evidencias que indican que los alumnos aprenden y retienen mejor los procesos, y la información en general, si se involucran en actividades de aprendizaje que requieren que ejecuten y piensen la información más que concentrarse en los procesos mecánicos de cálculo (Vellerman \& Moore, 1996). Esto se ve favorecido si el proceso de aprendizaje se apoya en la tecnología computacional -en este estudio se empleó el programa estadístico SPSS versión 17.0. Estos autores también señalan la importancia de aprender estadística empezando por las estrategias y herramientas necesarias para la exploración y comprensión de los datos. Además, se ha detectado el desfase entre los conceptos teóricos y la interpretación de éstos. No obstante, hay cuestiones que requieren solución desde la didáctica de la estadística. 
En cuanto a las estrategias para enseñar estadística, hay ciertas críticas que son generales a la enseñanza pero que son particularmente importantes en la enseñanza de la estadística: el aprendizaje de cuestiones declarativas abstractas, descontextualizadas y de relevancia cognitiva limitada. Se identifica de este modo una brecha entre el saber qué (know what) y el saber cómo (know how). Esto ha conducido a generar escenarios didácticos que potencien las capacidades de los alumnos para razonar estadísticamente en escenarios auténticos (de la vida real) -lo que puede mejorar a través de la relevancia cultural mediante problemas y ejemplos relevantes a las culturas a las que pertenecen los alumnos- y la actividad social, generando un escenario de prácticas colaborativas de solución de problemas debidamente mediadas por el docente.

Batanero (2001) argumenta que la estadística, a pesar de contar con una axiomática satisfactoria, es quizás la única rama de las matemáticas donde prosiguen hoy día las discusiones sobre la interpretación de conceptos básicos. Esta controversia no es de tipo técnico, ya que desde el punto de vista matemático, cualquier concepto estadístico queda determinado por su definición. La autora agrega que si el profesor no es consciente de esta problemática, difícilmente podrá comprender algunas dificultades de sus estudiantes, que necesitan materializar en ejemplos concretos los conceptos y modelos matemáticos.

Uno de los enfoques didácticos que se ha trabajado en la enseñanza de la estadística es el enfoque constructivista (Batanero, 2001) que recurre al uso de recursos informáticos para facilitar su aprendizaje a través de estrategias como: la interacción con objetos, la resolución de problemas, la interacción social y cultural, o mediante una combinación de las anteriores.

Los nuevos enfoques de la enseñanza de la estadística revelan que las mejores situaciones son aquellas donde los sujetos son llevados a construir por sí mismos las representaciones adecuadas (Shakarian, 1995; Lewis \& Williams, 1994; Cobb, 1993). Tal construcción activa parece ser un factor determinante de la estabilización de dichas representaciones. Batanero (2001) señala que al considerar la conveniencia o no de incluir un tema como objeto de enseñanza hay que tener en cuenta su utilidad y que este tema se halle al alcance de los alumnos.

Finalmente, y tomado de la didáctica de las matemática, se puede señalar la importancia y relevancia del enfoque ontosemiótico (Godino, Contreras \& Font, 2006) que permite la elaboración de configuraciones didácticas y su articulación en trayectorias didácticas, trayectorias epistémicas y trayectorias de mediación, haciendo central la construcción del conocimiento estadístico por los propios estudiantes. Al tener lugar en la clase y ser resultado de las interacciones de los alumnos con el medio creado por el profesor (del que también forma parte) tienen lugar procesos de reflexión e interpretación, tanto sobre los propios objetos estadísticos (situaciones, técnicas, etc.) como sobre los papeles a desempeñar por los distintos agentes. 


\section{Metodología}

\subsection{Procedimiento}

En la primera sesión se solicitó a los alumnos de posgrado que respondieran el Cuestionario de Actitudes hacia la Estadística (SATS-28). Se indicó que su participación no afectaría las notas que pudieren obtener en el módulo. Se aseguró que el instrumento se respondiera de manera anónima aunque se requirió que usaran un nombre ficticio. Al terminar el curso se les entregó el mismo cuestionario con instrucciones similares, incluyendo el nombre ficticio usado en el primer cuestionario.

Respecto a las estrategias de enseñanza se trabajó con la Teoría de las Configuraciones Didácticas de Godino et al. (2006). Se consideró las trayectorias del docente: distribución de las funciones docentes a lo largo del proceso de instrucción; y trayectorias del alumno: distribución de las funciones o roles desempeñados por los alumnos; trayectoria cognitiva: los significados personales de los alumnos, y trayectoria mediacional, distribución de los recursos tecnológicos utilizados. Los alumnos recibieron dos libros sobre análisis de datos, uno impreso y otro digital, además se les incluyó en un sitio creado específicamente para el desarrollo del módulo con material y ejercicios estadísticos en SPSS, aplicando las orientaciones de la didáctica de la estadística.

En relación a la trayectoria epistémica, se siguió el esquema planteado por los autores referidos en el párrafo anterior. Se definió lo situacional: se abordó el planteamiento de un ejemplar del tipo de problemas estadísticos; lo actuativo: se abordó el desarrollo o estudio de una manera de resolver determinados problemas estadísticos; lo lingüístico: se introdujeron notaciones, representaciones gráficas estadísticas; lo conceptual: se formularon, interpretaron y aplicaron definiciones de las unidades conceptuales u objetos de aprendizaje puestos en juego; lo proposicional: se enunciaron, interpretaron y aplicaron propiedades estadísticas; y lo argumentativo: se justificaron las acciones adoptadas o las propiedades enunciadas para abordar una situación y/o problema estadístico.

La definición de las trayectorias epistémicas permitió diseñar y poner en funcionamiento la práctica de la estadística con datos de investigaciones reales, empleando estadística descriptiva y estadística inferencial.

En el sitio web los alumnos pudieron examinar diferentes mapas conceptuales para el procesamiento estadístico, escuchar audios y ver videos, además de examinar abundantes ejemplos de investigaciones y sus respectivos procesamientos.

En la organización de las prácticas de los alumnos se trabajó con la metodología de proyectos que consideraron, por ejemplo, la estadística como herramienta de clasificación, comprensión de tablas, resúmenes estadísticos, asociación estadística, contraste de hipótesis, producción de resultados, razonamiento estadístico y narrativa estadística aplicando las normas APA. 
En el análisis de cada proyecto se consideraron los problemas de razonamiento estadístico y dificultades de aprendizaje de los alumnos de posgrado siguiendo la línea y desarrollo argumental de Batanero (2001).

\subsection{Participantes}

Participaron cuarenta y ocho alumnos en el desarrollo del módulo de estadística aplicada a la investigación, todos provenientes de una universidad pública. Los participantes fueron abogados, psicólogos, docentes de tres maestrías en ciencias sociales, con una edad promedio 43,72 años (D.E. $=6,46)$. Un $33,3 \%$ de los alumnos (16) de sexo femenino y un $66,6 \%$ (32) de sexo masculino.

\subsection{Método}

Se realizó un estudio cuantitativo para investigar el efecto en las actitudes de los alumnos de posgrado hacia la estadística a partir del desarrollo de un módulo de estadística aplicada a la investigación basado en la Teoría de las Configuraciones Didácticas y en la didáctica de la estadística de Batanero (2001), que involucró conceptos, problemas y análisis e interpretación estadística. Se empleó el cuestionario SATS-28 para el proceso de medición, dicha escala incluye 28 ítems. Los sujetos respondieron a una escala tipo Likert de siete puntos para juzgar cada ítem (en total desacuerdo: 1, ni acuerdo ni desacuerdo: 4, totalmente de acuerdo: 7). Las puntuaciones para cada subescala se determinaron sumando los valores de los ítems de la subescala y dividiéndolos por el número de ítems de la misma. Se revirtieron los valores en los ítems redactados de manera negativa antes de determinar los puntajes de la subescala.

Este cuestionario se aplicó al comienzo del semestre y al final del desarrollo del módulo. Cada alumno fue examinado en cuatro áreas: (a) Afecto - sentimientos de preocupación positivos y negativos; (b) Competencia cognitiva - actitudes hacia el conocimiento y habilidades intelectuales implicadas en la estadística; (c) Valor - actitudes hacia la utilidad, relevancia y valor de la estadística en la vida personal y profesional; y (d) Dificultad actitudes hacia la dificultad de la estadística como materia.

Cuadro 1. Escala de actitudes hacia la estadística (SATS-28)

\begin{tabular}{|l|c|l|}
\hline \multicolumn{1}{|c|}{ Subescala } & No. de ítems & \multicolumn{1}{c|}{ Ejemplo de ítems positivos y negativos } \\
\hline Valor & $\mathbf{9}$ & $\begin{array}{l}\text { Utilizo la estadística en la vida cotidiana (+) } \\
\text { La estadística no sirve para nada (-) }\end{array}$ \\
\hline Dificultad & $\mathbf{7}$ & $\begin{array}{l}\text { Me siento seguro cuando hago problemas de estadística (+) } \\
\text { La estadística es una asignatura complicada (-) }\end{array}$ \\
\hline Afecto & $\mathbf{6}$ & $\begin{array}{l}\text { Me gusta la estadística (+) } \\
\text { Me siento frustrado al hacer pruebas estadísticas (-) }\end{array}$ \\
\hline $\begin{array}{l}\text { Competencia } \\
\text { cognitiva }\end{array}$ & $\mathbf{6}$ & $\begin{array}{l}\text { Puedo aprender estadística (+) } \\
\text { Me resulta difícil comprender los conceptos estadísticos (-) }\end{array}$ \\
\hline
\end{tabular}


La actitud hacia la estadística se operacionalizó a partir de la puntuación total obtenida en la escala, y de las puntuaciones obtenidas en los diferentes componentes que conforman la escala: valor, dificultad, afecto y competencia cognitiva.

Cuadro 2. Composición de la escala

\begin{tabular}{|l|l|}
\hline \multicolumn{1}{|c|}{ Componentes } & \multicolumn{1}{|c|}{ Ítems } \\
\hline Afecto & $1,2,11,14,15,21$ \\
\hline Cognitivo & $3,9,20,23,24,27$ \\
\hline Valor & $5,7,8,10,12,13,16,19,25$ \\
\hline Dificultad & $4,6,17,18,22,26,28$ \\
\hline
\end{tabular}

Se calcularon las medias del pretest y postest para las cuatro subescalas y se aplicó la prueba $t$ Student para datos emparejados al final del semestre con el propósito de examinar si hubo cambios significativos en los puntajes pre-test y pos-test entre cualquiera de las observaciones.

Hipótesis: Los puntajes promedio en la Escala de actitudes hacia la estadística (SATS-28) al comienzo y al final para los alumnos de posgrado que participaron en las actividades de un módulo de estadística aplicada a la investigación, no serían significativamente diferentes al nivel $=0.05$.

Se procedió a estudiar las características de la escala porque para analizar los ítems, hacer algún grado de inferencia y evaluar la hipótesis, se requiere que la escala sea confiable. Se obtuvo un valor alfa $=0,7982$ para el coeficiente de Cronbach de la escala total. Se consideró un valor apropiado para los propósitos de esta investigación. Respecto a la confiabilidad de cada una de las subescalas, los resultados fueron los siguientes:

Cuadro 3. Confiabilidad de las subescalas

\begin{tabular}{|l|c|c|}
\hline \multicolumn{1}{|c|}{ Subescala } & Esta investigación & Otras investigaciones \\
\hline Valor & 0,81 & $0,74-0,90$ \\
\hline Dificultad & 0,79 & $0,64-0,81$ \\
\hline Afecto & 0,80 & $0,80-0,89$ \\
\hline Competencia cognitiva & 0,76 & $0,77-088$ \\
\hline
\end{tabular}

\section{Resultados}

Al examinar los resultados se pudo identificar que losítems con peores puntuaciones globales corresponden a los aspectos relacionados con la dificultad que implica el aprendizaje de la estadística, en especial el ítem la estadística implica mucho cálculo (media $=2,21$ ). Los ítems con mejores puntuaciones medias globales fueron puedo aprender estadística (media $=6,10)$, la estadística no sirve para nada, ítem negativo y por el cual se invierte la 
puntuación obtenida, arrojando una media igual a 5,95 que refleja alta valoración de parte de los alumnos. El resultado de este ítem se ve apoyado por los resultados de ítems tales como los conceptos estadísticos no se aplican fuera del trabajo (media $=5,67$ ). Examinada la variabilidad de los ítems (acuerdo/desacuerdo en la valoración), es interesante destacar que la dispersión menor de todos los ítems es 0,88 y corresponde al ítem que obtuvo mejor media: puedo aprender estadística. Un ítem que presenta una más alta dispersión es me da miedo la estadística $(\mathrm{s}=1,58)$ representando una posición más diferenciada.

A efectos de responder la pregunta investigativa y examinar la hipótesis, se usó la prueba estadística t-test que se usa para comparar los puntajes promedio obtenidos en el pretest y el postest en las cuatro subescalas del cuestionario SATS-28.

El cuadro 4 muestra los puntajes promedio y las desviaciones estándar para las cuatro subescalas del SATS-28. Además, se incluyen los valores t para muestras emparejadas y el nivel de significación (dos colas) para cada subescala. Como puede observarse en este cuadro, se obtuvo una diferencia significativa $(\mathrm{p}<0,05)$ en los puntajes promedio de la escala en el pre y post test para cada una de las cuatro escalas.

Los resultados de la prueba t-test indican que hubo diferencias estadísticamente significativas entre los puntajes promedio del SATS-28 del pretest y postest al 0,05 nivel de significación. Usando $\mathrm{a}=0,05$ como nivel de prueba se detectó suficiente evidencia para refutar la hipótesis nula respecto de las diferencias en la medición de los puntajes promedio en el cuestionario de actitudes hacia la estadística.

Cuadro 4. Puntajes promedio y desviaciones estándar para las cuatro subescalas del SATS28

\begin{tabular}{|l|c|c|c|c|c|c|}
\hline \multirow{2}{*}{ Escala } & \multicolumn{2}{c|}{$\begin{array}{c}\text { Pre-test } \\
\text { Puntajes Escalas }\end{array}$} & \multicolumn{2}{c|}{$\begin{array}{c}\text { Pos-test } \\
\text { Puntajes Escalas }\end{array}$} & \multicolumn{2}{c|}{$\begin{array}{c}\text { Diferencias Pos-Pre } \\
\text { Puntajes Escalas }\end{array}$} \\
\hline Afecto & 2,3 & 1,8 & 5,2 & 1,2 & 2,53 & $0,016^{*}$ \\
\hline Cognitivo & 2,9 & 2,3 & 5,7 & 1,4 & 3,07 & $0,025 *$ \\
\hline Valor & 3,1 & 2,1 & 5,8 & 1,2 & 3,03 & $0,024 *$ \\
\hline Dificultad & 4,9 & 2,8 & 2,3 & 0,9 & 2,63 & $0,018 *$ \\
\hline
\end{tabular}

\section{Discusión y conclusión}

Existen diversos estudios dirigidos a identificar los factores que afectan los procesos de aprendizaje cuando se incluyen contenidos estadísticos en los cursos de posgrado. Los resultados de esta investigación revelan que las percepciones negativas iniciales de los alumnos mejoraron después del período de mediación pedagógica a través de la aplicación de la Teoría de las Configuraciones Didácticas y Teoría de Situaciones Didácticas y el enfoque didáctico de Batanero (2001). No obstante, este resultado significativo debe ser 
precisado en términos de los factores que podrían haber actuado, por ejemplo, la modalidad del discurso del docente (actos de habla, tipos de conocimiento), la modalidad de las mediaciones, los trayectos epistémicos, las disposiciones de los alumnos, entre otros.

Se puede argumentar que para mejorar sus actitudes, los alumnos de posgrado requieren desarrollar tanto habilidades generativas como interpretativas. Por ejemplo, habilidades para comunicarse estadísticamente, habilidades de procedimientos, entender la lógica de los procesos estadísticos, análisis exploratorio de datos y la lógica de las investigaciones estadísticas (Gal \& Garfield, 1997; Batanero, 2001). Estas cuestiones se tuvieron presente en el desarrollo del módulo de estadística aplicada a la investigación, examinado en este estudio.

El puntaje promedio de los alumnos de posgrado que participaron en la mediación didáctica mediante el módulo de estadística aplicada a la investigación, reflejó actitudes más positivas al final que al inicio, en todas las sub-escalas. Esto implicaría que el desarrollo de la estrategia didáctica usada podría ser uno de los factores que produjo actitudes más positivas hacia el aprendizaje de la estadística y el análisis estadístico, facilitando los procesos de aprendizaje del módulo.

Respecto a la hipótesis investigativa, los puntajes promedio en el cuestionario SATS28 al comienzo y al final para los alumnos de posgrado que participaron en actividades didácticas que involucraron conceptos, problemas, análisis e interpretación estadística, serían significativamente diferentes al nivel $=0.05$, por consiguiente se refutó la hipótesis nula.

El proceso de análisis de los resultados también indicó que los factores dificultad y competencia cognitiva aparecen relacionados entre sí, lo mismo con el factor afecto. Se ha verificado empíricamente la relación entre lo cognitivo y afectivo. Una muestra de mayor tamaño permitiría examinar mejor la diferenciación de las actitudes. Por otra parte, se podría indagar otros componentes que pudieran estar presentes en la diferenciación de las actitudes.

Cuando se investiga en educación se deben considerar múltiples variables que podrían estar asociadas a los procesos de enseñanza-aprendizaje. Esto habría que realizarlo usando técnicas de análisis multivariante de datos, como el análisis factorial, el análisis de conglomerados y el escalamiento multidimensional. A futuro, se estudiará la estructura de cuatro factores del SATS, que si bien ha sido verificada por distintos investigadores, requeriría ser examinada con nuevas muestras y con sujetos de diferentes campos culturales. Esta investigación revelaría la necesidad de una mayor comprensión de las actitudes de los alumnos de posgrado hacia la estadística y su inter-relación con el logro y uso en el mundo real, y una didáctica más apropiada -por ejemplo, la de Godino, Contreras y Font (2006)para generar aprendizajes más significativos y actitudes más positivas de parte de alumnos y profesores.

Por otra parte, se requeriría mejorar las habilidades de comprensión matemática y reducir el nivel de ansiedad hacia la disciplina. La instrucción efectiva de la estadística necesita 
sustentarse en la comprensión de los conceptos estadísticos básicos y en la construcción progresiva de representaciones mentales implícitas o explícitas, como señala Vergnaud (1990 citado por Godino, 1991).

Adicionalmente, es necesario un sustento teórico de la didáctica para enseñar la estadística. Se podría pensar que la Teoría de la Configuraciones Didácticas (Godino, Contreras \& Font, 2006) y la Teoría de Situaciones Didácticas (Godino, 1991) pueden ser un camino apropiado. Se entiende por una situación didáctica un conjunto de relaciones explícita y/o implícitamente establecidas entre un alumno o un grupo de alumnos, algún entorno (incluyendo instrumentos o materiales) y el profesor con el fin de permitir a los alumnos aprender -esto es, reconstruir- algún conocimiento.

Para que el alumno construya el conocimiento, es necesario que se interese personalmente por la resolución del problema planteado en la situación didáctica. En este caso se dice que se ha conseguido la devolución de la situación al alumno. Por ende, un escenario didáctico con adecuadas mediaciones ofrecería mayores oportunidades para generar aprendizajes significativos y auténticos.

Finalmente, es importante señalar que en la actualidad se está trabajando en ciertas cuestiones teóricas para el estudio de las actitudes. Teóricamente, el estudio de las actitudes implica que sus configuraciones, formas de organización y categorización sean fundamentalmente de carácter histórico-cultural, lo cual significa entenderlas en el contexto de la actividad de sujetos y campos culturales específicos. Se trata de entenderlas en su historicidad, vinculadas a creaciones culturales colectivas y no a esquemas mentales aislados. Las actitudes actúan como elementos de estructuración de discursos sobre personas, sucesos, objetos, proporcionando una dimensión evaluativa desde la cual se producen los procesos de significación personal y cultural.

Metodológicamente se tendrían que estudiar los actos de habla de los profesores de estadística en la clase y los tipos de conocimientos a los que éstos se refieren. Este tipo de exploración se haría desde la dimensión pragmática del discurso de los docentes de estadística y desde una perspectiva lingüística, específicamente se realizaría un análisis del discurso (Van Dikj, 1980). Desde la perspectiva de los alumnos de posgrado, se debe trabajar con las representaciones sociales que tienen los estudiantes de posgrado sobre la estadística. De este modo se podría examinar la hipótesis sobre la posible relación entre actitudes y representaciones sociales. Para este efecto, se empleará la técnica de las redes semánticas naturales (Valdez, 1998). Constituye una técnica para evaluar el significado que se le asigna a un concepto y la idea es disponer de un procedimiento que posibilite obtener redes naturales, esto es, redes generadas por los propios sujetos, puesto que se prioriza la perspectiva del sujeto investigado, su propio lenguaje, como medio de acercarse al conocimiento de la subjetividad y entenderla de manera contextualizada. Se parte del supuesto básico de que las ideas que los sujetos tienen sobre los objetos no están en el vacío sino que se encuentran relacionadas con un contexto determinado. 


\section{Referencias bibliográficas}

Batanero, C. (2001). Didáctica de la estadística. Grupo de Investigación en Educación Estadística. Departamento de Didáctica de la Matemática. Universidad de Granada. Recuperado el 23 de abril de 2004 de http://www.ugr.es/ batanero/publicaciones.htm

Blanco, A. (2008). Una revisión crítica de la investigación sobre las actitudes de los estudiantes universitarios hacia la Estadística. Revista Complutense de Educación, 19 (2), 311-330.

Bradstreet, T. (1996). Teaching Introductory Statistics Courses so that Non Statisticians Experience Statistical Reasoning. American Statistician, 50 (1), 69-78.

Cobb, G. (1993). Reconsidering Statistics Education: A National Science Foundation conference. Journal of Statistics Education, 1 (1). Recuperado el 12 de mayo de 2008 de http://www.stat.unipig.it/ncsu/info/jse/v1n1/cobb.html

Eagly, A. H. \& Chaiken, S. (Eds.). (1993). Attitude structure and function. New York: McGraw Hill.

Gal, I. \& Garfield, J. B. (Eds.). (1997). The Assessment Challenge in Statistics Education. Amsterdam: IOS Press.

Gal, I. \& Ginsburg, L. (1994). The role of beliefs and attitudes in learning statistics: Towards an assessment framework. Journal of Statistics Education, 2 (2), 1-16.

Gal, I., Ginsburg, L. \& Schau ,C. (1997). Monitoring attitudes and beliefs in statistics education. En I. Gal \& J. B. Garfield (Eds). The assessment challenge in statistics education (pp. 37-51). Netherlands: IOS Press.

Godino, J. D. (1991). Hacia una teoría de la didáctica matemática. En A. Gutiérrez (Ed.). Área de Conocimiento: Didáctica de la Matemática (pp. 105-148). Madrid: Síntesis.

Godino, J. D., Contreras, A. \& Font, V. (2006). Análisis de procesos de instrucción basado en el enfoque ontológico-semiótico de la cognición matemática. Recherches en Didactique des Mathématiques, 26 (1), 39-88.

Keeler, C. \& Steinhorst, K. (1995). Using Small Groups to Promote Active Learning in the Introductory Statistics Course: A Report from the Field. Journal of Statistics Education 3 (2). Recuperado el 15 de mayo de 2008 de http://www.amstat.org/publications/JSE/ v3n2/keeler.html

Kirk, R. E. (2002). Teaching Introductory Statistics: Some Things I Have Learned, paper presented at the Annual Conference of the American Psychological Association, Chicago, IL. (ERIC Document Reproduction Service No. ED 473 611).

Lewis, L. H. \& Williams, C. J. (1994). Experiential learning: Past and present. En L. Jackson \& R. S. Caffarella (Eds.). Experiential learning: A new approach (pp. 5-16). San Francisco: Jossey-Bass.

Romero, R., Ferrer, A., Capilla, C., Zunica, L., Balasch, S., Serra, V. et al. (1995). Teaching Statistics To Engineers: An Innovative Pedagogical Experience. Journal of Statistics Education (online) 3(1). Recuperado el 15 de mayo de 2008 de http://www.amstat.org/ publications/jse/v3n1/romero.html

Schau, C., Stevens, J., Dauphine, T. L., \& Del Vecchio, A. (1995). The development and validation of the Survey of Attitudes Towards Statistics. Educational and Psychological Measurement, (55), 868-875.

Schutz, P. A., Drogosz, L. M., White, V. E., \& Distefano, C. (1998). Prior knowledge, attitude, and strategy use in an introduction to statistics course. Learning and Individual 
Differences, (10), 291-308.

Shakarian, D. C. (1995). Beyond lecture: Active learning strategies that work. Journal of Physical Education, Recreation, and Dance, 66, (May/June), 21-24.

Snee, R. (1993). What's Missing in Statistical Education. American Statistician, 47 (2), 149154.

Sorge, C. \& Schau, C. (2002, abril). Impact of engineering students' attitudes on achievement in statistics: a structural model. Documento presentado en la Annual Meeting of the American Educational Research Association, New Orleans.

Valdez, J.L. (1998). Las redes semánticas naturales, usos y aplicaciones en la psicología social. México: Universidad Autónoma del Estado de México.

Van Dikj, T. (1980). Estructuras y funciones del discurso. México: Siglo XXl.

Vellerman, P. F. \& Moore, D. S. (1996). Multimedia for teaching statistics: Promises and pitfalls. The American Statistician, (50), 217-225.

Watts, D. (1991). Why is Introductory Statistics Difficult to Learn? And What Can We Do to Make it Easier? The American Statistician ,45 (4), 290-291. 